\title{
DIDÁCTICA PARA LA FORMACIÓN EN FINANZAS EN PROGRAMMAS UNIVERSITARIOS DE ADMINISTRACIÓN DE EMPRESAS
}

\section{DIDACTICS FOR TRAINING IN FINANCE IN UNIVERSITY PROGRAMS OF BUSINESS ADMINISTRATION}

\author{
Florentino Rico-Calvano ${ }^{1}$
}

\section{RESUMEN}

El capítulo es resultado de la tesis doctoral Didáctica para la formación en finanzas en programas universitarios de Administración de Empresas desde una perspectiva constructivista. La investigación es abordada bajo el enfoque cuantitativo y el paradigma empírico analítico, investigación descriptiva-explicativa. Se identificaron, caracterizaron e indagaron los trabajos de los docentes de finanzas en las diferentes universidades desde los estilos de enseñanza, didácticas, actividades del aprendizaje, contenidos, sistemas de evaluación, motivación, currículo y pedagogía. Se hizo triangulación de datos, teorías, metodologías, disciplinar y del investigador para la nueva construcción del conocimiento didáctico para llegar a la propuesta de una Didáctica dinámica en contexto fundamentada en las concepciones epistemológicas, filosóficas y categorías de la investigación: didáctica, currículo, formación, pedagogía y finanzas. Se concluyó con la propuesta didác-

\footnotetext{
Capítulo derivado del proyecto de investigación "Didáctica para la formación en finanzas en programas universitarios de Administración de Empresas". Trabajo de grado del Doctorado en Ciencias de la Educación de la Universidad Simón Bolívar durante los años 2013-2016.

1 Universidad Simón Bolívar. Barranquilla-Colombia.

florentinorico@unisimonbolivar.edu.co - https://orcid.org/0000-0002-6477-3761

Doctor en Ciencias de la Educación. Investigador Asociado Senior y líder del grupo de investigación Democracia y Modernización del Estado Colombiano de la Universidad Simón Bolívar de Barranquilla. Economista. Becario OEA y BID. Experto en Formulación y Evaluación de Proyectos. Gestión Gerencial. Especialista en Política Fiscal y Administración Financiera Tributaria. Categoría $\mathrm{A}_{1}$.

florentinorico03@hotmail.com
} 
tica dinámica en contexto del proceso enseñanza-aprendizaje, cuyos actores principales son docentes y estudiantes, la secuenciación del proceso didáctico para la formación en finanzas se halla aislada de los problemas cotidianos de la economía. El administrador del siglo XXI debe ser crítico, capaz de prevenir y solucionar problemas, trabajar en grupo, sin perder de vista que el conocimiento por sí solo no es útil, cobra utilidad en términos de innovación y transformación solamente cuando se pone al servicio de la sociedad.

Palabras clave: Didácticas, Formación, Finanzas, Pedagogía y Currículo.

\section{ABSTRACT}

The chapter is the result of the doctoral thesis Didactics for training in finance in university programs of Business Administration from a constructivist perspective. The research addressed under the quantitative approach and the empirical analytic paradigm, descriptive-explanatory research. They were identified, characterized and investigated the work of finance teachers in different universities from the styles of teaching, didactics, learning activities, content, evaluation systems, motivation, curriculum and pedagogy. It was done triangulation of data, theories, methodologies, disciplinary and of the researcher for the new construction of the didactic knowledge to arrive at the proposal of a didactic dynamic in context based on the epistemological, philosophical and research categories: didactics, curriculum, training, pedagogy and finance. It was concluded with the dynamic didactic proposal in the context of the teaching-learning process, whose main actors are teachers and students, the sequencing of the didactic process for training in finance is isolated from the daily problems of the economy. The manager of the 21st century must be critical, capable of preventing and solving problems, working in groups, without losing sight of the fact 


\section{Didáctica para la formación en finanzas en programas}

universitarios de Administración de Empresas

that knowledge alone is not useful, becomes useful in terms of innovation and transformation only when it is put to the service of the society

Keywords: Didactics, Training, Finance, Pedagogy and Curriculum.

\section{INTRODUCCIÓN}

Los cambios en la sociedad implican transformar la educación, la enseñanza centrada en los estudiantes, los entornos concentrados en el conocimiento. Hoy los nuevos escenarios son originados por los cambios estructurales: corrupción, crisis mundial, globalización, competitividad, desequilibrios de las finanzas; coyunturales: cómo recuperar el crecimiento económico, la confianza, gobernabilidad, incremento de la inversión privada, el dilema entre inflación, desempleo, crecimiento y recesión económica, monitorear los planes de desarrollo, la asignación eficiente de los recursos para alcanzar la promoción y el desarrollo sostenibles con estabilización económica, enfrentar los retos de la economía mundial; que han generado procesos inciertos y complejos como la caída de los precios del petróleo que afectan crecimiento, recaudo y frenos en los proyectos de inversión, menor crecimiento económico, aumento del tipo de cambio, fluctuaciones de las tasas de interés y desempleo. A esto se agrega el proceso de paz que demanda una serie de recursos financieros que obliga a la formación en finanzas en todas las disciplinas para el manejo de los pocos recursos disponibles, sean propios o de terceros.

Hoy las universidades miran el pasado con algo de gratitud, pero el futuro es el reto para enfrentar las nuevas tendencias de la tecnología y las prácticas educativas, es por ello que la búsqueda de alternativas que optimicen la formación de sus estudiantes que cursan sus carreras universitarias es la misión y visión en sus proyectos educativos insti- 
tucionales. Se busca que la didáctica que se utiliza en la formación en finanzas en los programas universitarios de Administración de Empresas responda al contexto económico y financiero y a las expectativas del estudiante que aplica la asignatura de Finanzas como la encargada de administrar el dinero.

Las universidades modernas aúnan esfuerzos en la revisión de sus currículos, planes de estudios y programas para actualizarlos y ajustarlos a la coyuntura y normas establecidas en materia educativa en créditos, currículo (propósitos, contenidos, secuenciación, métodos, resultados y evaluación), pedagogías, didácticas, y estilos de enseñanza. Ajustes que permiten superar cualquier vacío que experimente el estudiante y egresado en su actividad profesional.

Se pretende como objetivo generar lineamientos didácticos dinámicos en contexto para la formación en finanzas en programas universitarios de Administración desde una perspectiva constructivista que permita favorecer el proceso de enseñanza-aprendizaje de los estudiantes para superar las diferencias entre la teoría y la práctica en la enseñanza de las finanzas y fundamentar desde el saber didáctico su resignificación para la formación en finanzas de los administradores de empresas cimentado en un proceso de transformación de la pedagogía tradicional hacia la pedagogía activa y cognoscitiva.

El análisis se inició con el planteamiento del problema, la problemática existente, sus causas y efectos, se identificó el problema, se fijaron los objetivos y alcance de la investigación; se presentó el diseño metodológico, en el cual se mostró la metodología que se utilizó para el desarrollo y culminación del trabajo basada en el enfoque constructivista, la fundamentación teórica mostró la fundamentación teórico-conceptual que sirvió de base a las ideas que se presentaron desde el abordaje 
de las diferentes teorías, desde el estado del arte con sus referentes correspondientes, las conclusiones y recomendaciones y la propuesta didáctica dinámica en contexto para la formación en finanzas.

\section{ESTADO DEL ARTE Y FUNDAMENTACIÓN TEÓRICA DE LA DIDÁCTICA EN LAS FINANZAS}

\section{Didáctica}

La didáctica es la primera disciplina pedagógica que se constituye en un cuerpo de doctrina autónoma, cuyos precursores según García (1969), son el teólogo alemán Hugo de San Víctor (1096-141), Vives (1492-1540), Ratke o Ratichius (1571-1635) y Alsted (1588-1638). Luego sigue la didáctica científica-esencialista, etapa superior con el pensador checo moravo Juan Amós Comenio en su obra Didáctica Magna, publicada en 1657. A partir de Comenio, la didáctica es visualizada como una técnica o serie de prácticas a ser ejecutadas por los docentes. Posteriormente, la didáctica y metodología psicológica se presentaron en esta etapa como una evolución histórica de profundización de la orientación naturalista-esencialista. Se destaca la doctrina herbartiana que se desarrolló en la segunda mitad del siglo XIX y coincide con ella la incorporación a la pedagogía moderna de la investigación experimental que hizo sus aportes en la formación en finanzas con la articulación entre didáctica, pedagogía y currículo.

Finalmente la etapa moderna-experimental, que se caracteriza por el desarrollo de métodos y de manera gradual se pasa de los verbales a los intuitivos y de estos a los activos; estos últimos son la culminación del desarrollo psicológico al servicio de la enseñanza. Se destaca la presencia del ruso Sikorsky, quien publica un trabajo sobre la fatiga que el trabajo intelectual produce en los escolares (García, 1969). 
Es interesante abordar algunas conceptualizaciones sobre la didáctica presentadas por García (1969), cuando afirma que todas las cosas que se enseñan son muy valiosas y tienen una metodología de tipo artístico. Hay quienes opinan de manera clara que la didáctica es arte, centrado en el carácter del maestro y en el enfoque de la didáctica en cuanto a la enseñanza.

La experiencia ha demostrado que en la formación en finanzas, la didáctica es como un don magistral que no se puede transmitir ni regular de ninguna forma; es el arte no transferible por la destreza, habilidades y malicia indígena que debe asumir el administrador de empresas en el proceso administrativo moderno.

Martínez (2007) sostiene que la didáctica "es el arte de saber transmitir los conocimientos de manera adecuada para su asimilación" (p.32) planteamiento que permite enfocarla en la acumulación de conocimiento.

El debate contemporáneo sobre didáctica la divide en didáctica general y específica. Díaz (1999) ve la didáctica general "como la ciencia que explica y aplica lo relacionado con la enseñanza como tarea intencional, sistemática y como estrategia expedita para logar la formación del hombre"(pp.108-110). La didáctica específica tiene en cuenta los campos de conocimiento, los niveles de educación, edades, tipos de establecimientos y características de los sujetos. El conocimiento científico denominado saber sabio va de lo implícito a lo explícito y es adaptado por la transposición didáctica cómo saber enseñar finanzas, es transformado en objeto de enseñanza. La teoría la proporciona la pedagogía que es la ciencia de la educación y la práctica, es decir, el cómo hacerlo, lo proporciona la didáctica. 
La didáctica es el campo disciplinar de la pedagogía que se ocupa de la sistematización e integración de los aspectos teóricos metodológicos del proceso de comunicación que tiene como propósito el enriquecimiento en la evolución del sujeto implicado en este proceso. De acuerdo a Aguilar (2004), se agrega que la didáctica es el arte de saber explicar y enseñar con mayores recursos para que el alumno entienda y aprenda según lo planteado por Ramírez (2013).

Concepto de didáctica: Ciencia de enseñar y aprender, de la enseñanza, de los contenidos formativos, de los procesos de aprendizaje y de aplicación, de las teorías psicológicas de la enseñanza-aprendizaje con principios, leyes, marcos teóricos y metodológicos que modelan creativamente la intervención pedagógica en el escenario académico.

\section{Finanzas}

Ciencia aplicada de la economía, se encarga de la efectividad de la administración del dinero, dentro del equilibrio entre riesgo, liquidez y rentabilidad. La finanza hace parte de la economía y surge como campo de estudio independiente a principios del siglo XX; se relaciona con la contabilidad, generadora de reportes financieros e insumo de las finanzas. Hoy la finanza se resume en administrar dinero.

A comienzos del siglo XX empezaron a surgir las asociaciones de contadores que además de sus propias normas establecieron convenios y pautas para el ejercicio de las actividades contables en Suiza en 1916 y en Japón en 1917. Como bases para el ejercicio de la finanza, en 1934 surgieron las primeras seis reglas de los principios contables de hoy, en los años 1936, 1941, 1948 y 1957 se publicó y adicionó por parte de la American Acounting Association (AAA), todo lo relacionado con los principios contables (Sinisterra, 2001). Con la crisis en 1930, la finanza 
se hace necesaria para la supervivencia y la preservación de la liquidez, entonces la preocupación era protegerse, predominando el criterio conservador de mantener una estructura financiera sólida.

La finanza estudia decisiones administrativas para adquisición y financiamiento de activos circulantes y fijos, estructura de los pasivos, los problemas de tamaño y crecimiento de la empresa (Philippatos, 1974). Otros autores definen finanzas como la asignación de fondos dentro de la empresa y su adquisición (Van Horne, 1988). Las finanzas para efecto de esta investigación es la ciencia y arte de administrar dinero Gitman, 2000) (Ver Figura 1).

La finanza surge de la necesidad de la fusión del enfoque clásico y el operacional según la investigación realizada por Puche (2010), para su aplicación se demanda de una formación en la administración del dinero, como resultado de una didáctica que se articule con la pedagogía y el currículo para lograr el equilibrio en los signos vitales de las empresas de liquidez, rentabilidad y riesgo.

Los premios Nobel de Economía aportan a la formación en finanzas, numerosas investigaciones y conceptualizaciones con cierta polisemia de conceptos, pero en general giran sobre los conceptos básicos de liquidez, rentabilidad, riesgo y últimamente sobre balance scored. Se analizaron y articularon en la investigación sus aportes de manera cronológica desde 1969 hasta 2012, (Rico, 2013).

\section{Currículo}

El concepto de currículo tiene diferentes definiciones y existe cierta polisemia en cuanto a su significado. La complejidad del concepto de 
currículo ha sido ampliamente tratada por diversos autores que han intentado afrontar distintas conceptualizaciones. Para Mora (2014), el currículo es el instrumento que interioriza y moviliza las condiciones subjetivas y objetivas en un plan de estudios, desde las diferentes áreas del saber, asignaturas o proyectos pedagógicos institucionales, con la función de movilizar la intersección de estas estructuras con los sujetos de formación de acuerdo con la misión y visión de una institución educativa (Ver Figura 1).

Por otra parte, Eigenmann (1981) considera al currículo como un documento que proporciona al profesor la base para planificar su enseñanza, que contiene enunciado acerca de su uso, implica objetivos a conseguir a los que se subordinan los de contenidos de enseñanza estructurados y lo que involucra su continua evaluación y mejora.

Pero Stenhouse (1987) es amplio cuando desde su perspectiva didáctica mira el currículo como una tentativa de comunicar los principios y rasgos esenciales de un propósito educativo, de forma tal que permanezca abierto a la discusión crítica y pueda ser trasladado efectivamente a la práctica; plantea la necesidad de vincular teoría y práctica en el desarrollo del currículo.

El profesor Ossa (2013) sostiene que una "visión postmoderna del currículo es más un proceso de desarrollo que un cuerpo de conocimiento fijo y acabado que necesita ser cubierto o aprendido" y remata afirmando que "el currículo se refiere a todo lo que acontece en el proceso educativo" (p.8). El mapeo sobre el currículo es extenso con una gama de conceptualizaciones, destacándose significativos escritos de investigadores que han aportado un marco teórico valioso sobre currículo, como John Dewey, Ralph Tyler, Franklin Bobbit, William Kilpatrick, Benjamin Bloom, Demain, George S. Counts, Ibáñez y Sarramona (Ver Figura 1). 


\section{Formación}

En Colombia tomamos como referente a Zambrano (1963), quien plantea "que la especificidad de la educación es la razón, mientras que la de la formación es la experiencia y el devenir" (p.75), luego la educación entra a promover un sentimiento de transformación sobre el sentimiento.

Como un conjunto de prácticas culturales, sociales o económicas que expresan los discursos de la sociedad, una moral, las prácticas de poder y cuyo espacio son las profesiones y sus tecnologías de saber. El profesor se apropia las tecnologías de saber que circulan en los dispositivos de formación y reproduce las fabricadas por el discurso dominante y puestas a funcionar por ellos (Zambrano, 1963).

Ahora en la didáctica para la formación en finanzas es pertinente considerar lo planteado por Correa (2013), donde la identificación de los procesos didácticos, la educación y conocimiento, conocimiento y educación, son dos pilares sobre los que la humanidad fundamenta sus procesos transformadores, hábitos, valores, pautas culturales, prácticas, conductas, métodos, formas, contenidos, mitos, tradiciones, sueños, leyendas, ciencia, técnica, arte y cualquier forma del ser, hacer y pensar humano; se entrelaza, se religa con alguna manifestación de lo educativo en cualquiera de las perspectivas y contextos.

En Argentina, para Monetti (2015) “La formación aparece como elemento recurrente que en sus relaciones con otros componentes van tomando diversas formas, luego la define como un proceso de desarrollo personal de un sujeto que se forma a sí mismo por mediaciones, está ligada a las transformaciones identitarias que se producen en los estudiantes" (p.165). 


\section{Didáctica para la formación en finanzas en programas}

universitarios de Administración de Empresas

Según Aebli (2000), la formación es un proceso de construcción social de la realidad muy particular que configura un formar trabajando, construido y reconstruido a través del tiempo. La formación no puede abordarse aislada de los problemas financieros que viven la sociedad y la economía (Ver Figura 1).

\section{Pedagogía}

Debe plantear seriamente los problemas de las finanzas, de la comunicación y de la información del hombre como ser viviente en relación con la red de sistema y sus efectos sobre la cultura y las formas del poder saber. Para la formación en finanzas en la administración del dinero juega un rol importante el modelo de pedagogía activa y cognoscitiva.

Por lo tanto, la pedagogía relacionada con la didáctica para la formación en finanzas según Ontoria (2003), es una experiencia personal que permite construir conocimiento, en la cual interviene el yo con todas sus capacidades, emociones, sentimientos, motivaciones y habilidades.

Finalmente, Bernstein (1971) “sostiene que la práctica pedagógica es una forma de organización jerárquica, comunicativa, escolar, que se inscribe en un discurso pedagógico" (p.47).

La pedagogía es abordada desde la tendencia pedagógica con caracterización de cinco categorías: a) Un docente capaz de transformar su práctica didáctica en la formación en finanza, b) Un docente autocrítico de sus prácticas, de sus interacciones, de sus lógicas, con decisión e iniciativas concretas para transformarlas en la administración del dinero, c) Un docente que considere la historicidad del acto educativo, d) Un 
docente que no pierde la perspectiva interpretativa del conocimiento que nutre su práctica financiera y e) Un docente que considere al estudiante como un sujeto de su formación, constructor de conocimiento. Se agrega que el docente es el recurso didáctico más importante, mientras que Stenhouse (1987) lo eleva a la categoría de investigador en el aula (Ver Figura 1).
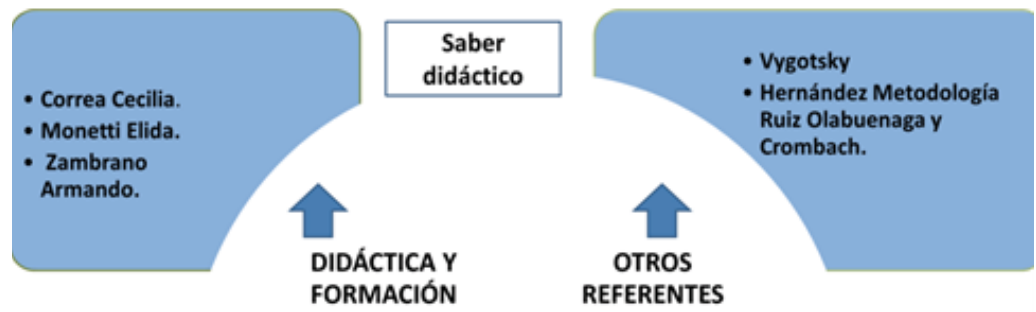

\section{MÉTODO}

La metodología utilizada se amarró al enfoque paradigmático cuantitativo y epistemológicamente en el paradigma empírico analítico con un tipo de investigación descriptivo-explicativo, considerando la posición de Hernández (2014, pp.35-50) y Galeano (2004) con nivel de investigación desde la perspectiva interpretativa. 
La población la define Chávez (2001) como el universo de la investigación en la cual se generalizaron los resultados, constituida por características o estratos que permitieron distinguir los sujetos, unos de otros. Para la población en estudio se tuvieron en cuenta los programas de Administración de Empresas de las universidades tomadas como muestra en el distrito de Barranquilla, que imparten actualmente la modalidad presencial. La población objeto de investigación fueron docentes de tiempo completo, medio tiempo, catedráticos y coordinadores, estudiantes de los programas universitarios de Administración de Empresas de las universidades seleccionadas en la muestra. Se tomaron 3 universidades del distrito de Barranquilla (10\% oficial y $20 \%$ privadas), el censo poblacional fue conformado por 215 sujetos, se utilizó el censo poblacional de 280 estudiantes de acuerdo a lo establecido por Tamayo y Tamayo (2001) por tratarse de una población pequeña y manejable, se aplicó la fórmula que determinó una muestra representativa de 191 estudiantes, 21 docentes y se hicieron 9 observaciones de clase a docentes de tiempo completo, medio tiempo y catedráticos seleccionados en el censo.

El objetivo básico del estudio fue describir la relación entre docentes y estudiantes en lo correspondiente a la didáctica para la formación en finanzas. Se entrevistaron por separado a cada uno de los actores y luego se correlacionaron sus respuestas con sus pares (estudiante y docentes); además mediante la observación de la clase de cada docente seleccionado en la muestra se apreciaron correlaciones en el proceso didáctico de las finanzas, tanto del docente como de los estudiantes.

Para extraer la información a la población se aplicó la técnica de la encuesta de tipo descriptiva que permitió recoger, procesar y analizar informaciones que resultaron de las unidades investigadas del colectivo determinado. Los datos fueron vaciados en una matriz de doble entrada y activados los valores con la fórmula de Alfa Cronbach. 


\section{Resultados, discusiones y conclusiones}

Resultados del desempeño académico de los docentes y estudiantes de los programas universitarios de Administración de Empresas de las universidades consideradas en la muestra.

\section{RESULTADOS DEL DESEMPEÑO ACADÉMICO DE LOS DOCENTES}

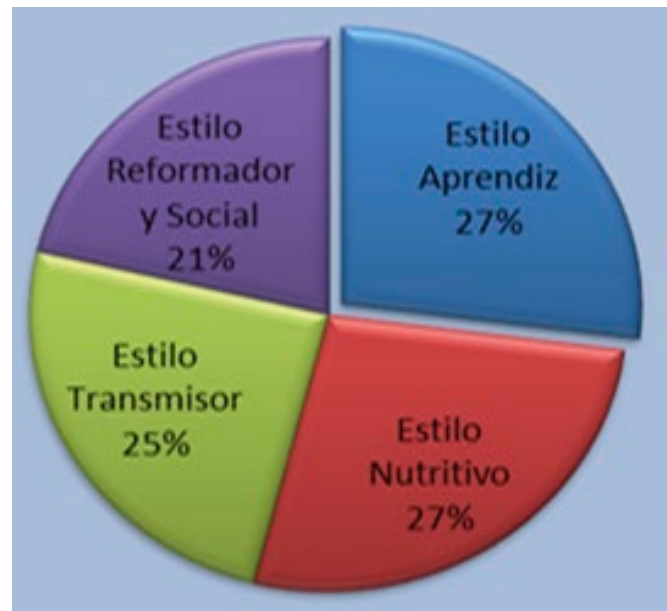

Figura 2. Estilos de enseñanzas de los docentes en los programas universitarios de Administración de Empresas encuestados de las universidades: 1023B-6542F-9783E2016 Fuente: (Rico, 2016)

Se identificó como destacada en la formación en finanzas el estilo de enseñanza Nutritivo que alcanzó en completamente y medianamente de acuerdo aceptación positiva del 83,8 \% (42,9\%+42,9\%), siguió en orden de preferencia el estilo Transmisor aplicado por los docentes que se mostraron completamente y medianamente de acuerdo en un $76,2 \%(57,2 \%+19,0 \%)$, estilo de enseñanza que se encuentra por encima del promedio del 72,15\% (Ver Figura 2).

Entre los tipos de didáctica que utilizan los docentes se identifica- 


\section{Didáctica para la formación en finanzas en programas}

universitarios de Administración de Empresas

ron como de mayor aplicación: la didáctica comprehensiva con el $85,7 \%$ discriminada en un $57,1 \%$ medianamente de acuerdo y un $28,6 \%$ completamente de acuerdo; sigue, la didáctica Crítica con el 76,2\% $(47,6 \%+28,6 \%)$ y la didáctica Expresiva, la Expositiva, la Constructivista, y la didáctica Socrática con el 71,4\% (Ver Figura 3).

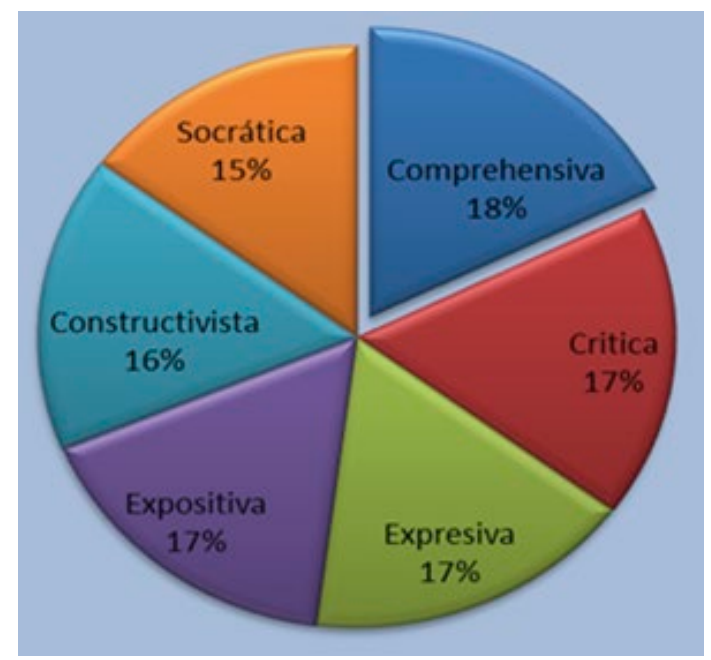

Figura 3. Tipos de didácticas utilizadas por los docentes en los programas universitarios de Administración de Empresas encuestados en las universidades: 1023B-6542F-9783E-2016 Fuente: (Rico, 2016)

En la consulta se caracterizó y evidenció que en los tipos de metodologías, el método de casos aplicados por los docentes alcanzó el 81,0 \%, seguido en su orden de importancia El método talleres teóricos y prácticos con el 80,9 \% de aplicación. El trabajo colaborativo o en equipo registró un 76,2 \% positivo en su aplicación por los docentes encuestados, mientras que los juegos financieros de bolsas de valores solo alcanzaron en su aplicación los niveles más bajos del 76,1\%, el promedio de aplicación de los métodos de enseñanza es del 78,55 \%, es decir, solo los métodos de casos y teóricos y de talleres lograron sobrepasar los promedios (Ver Tabla 1). 
Tabla 1

Métodos de enseñanza utilizados por los docentes entrevistados en los programas universitarios de Administración de Empresas- 2016

\begin{tabular}{|c|c|c|c|c|c|}
\hline Métodos de enseñanza & $\begin{array}{l}\text { Juegos finan- } \\
\text { cieros (Bolsa } \\
\text { de valores) }\end{array}$ & De casos & $\begin{array}{l}\text { Colaborativo } \\
\text { (trabajo en } \\
\text { equipo) }\end{array}$ & $\begin{array}{l}\text { Talleres teóri- } \\
\text { cos y prácticos }\end{array}$ & Promedio \\
\hline Variables & $\begin{array}{l}\text { Frecuencia } \\
\text { Relativa \% }\end{array}$ & $\begin{array}{l}\text { Frecuencia } \\
\text { Relativa \% }\end{array}$ & $\begin{array}{l}\text { Frecuencia } \\
\text { Relativa \% }\end{array}$ & $\begin{array}{l}\text { Frecuencia } \\
\text { Relativa \% }\end{array}$ & \\
\hline $\begin{array}{l}\text { Completamente en des- } \\
\text { acuerdo }\end{array}$ & 4,8 & 0 & 0 & 0 & \\
\hline $\begin{array}{l}\text { Medianamente en des- } \\
\text { acuerdo }\end{array}$ & 4,8 & 0 & 0 & 4,8 & \\
\hline $\begin{array}{l}\text { Ni de acuerdo, ni en } \\
\text { desacuerdo }\end{array}$ & 14,3 & 14,3 & 19,0 & 9,5 & \\
\hline Medianamente de acuerdo & 19,0 & 42,9 & 42,9 & 47,6 & 38,1 \\
\hline $\begin{array}{l}\text { Completamente de } \\
\text { acuerdo }\end{array}$ & 57,1 & 38,1 & 33,3 & 33,3 & 40,45 \\
\hline Total & 100,0 & 100,0 & 100,0 & 100,0 & \\
\hline
\end{tabular}

Fuente: Rico (2016) Tesis doctoral Didáctica para la formación en finanzas en programas universitarios de Administración de Empresas. Creatividad del investigador.

\section{Tipos de pedagogía y currículo utilizados}

El 90,5\% de los docentes resuelve, informa resultados y hace valoraciones individuales de las posibles dudas surgidas en las clases teóricas y prácticas para la realización de las actividades.

El 81,0 \% de los docentes consideraron que el curso de finanzas en la estructura curricular responde a la formación integral de un profesional para la toma de decisiones financiera en un contexto globalizado.

El $66,7 \%$ de los docentes encuestados insertaron en sus programas el componente de investigación, solo el 4,8 \% no aplicaron en la encuesta.

El 52,4\% de los docentes manifestaron que los créditos que tienen los cursos de finanzas en el plan de estudio son insuficientes como campo disciplinar para complementar la formación universitaria en Administración. 
Percepción de las actividades del aprendizaje desarrolladas por los docentes

Solo un 57,1 \% de los docentes son partidarios de que las actividades de aprendizaje deben ser variadas, múltiples, organizadas, secuenciales y temporizadas según un plan previo del docente, que a su vez responda a los objetivos y contenidos del curso.

El 81,0 \% de los docentes sostienen que sus actividades son evaluadas y supervisadas, mientras que el 76,2 \% dicen que sus actividades en el aula responden a los objetivos y contenidos del curso desarrollado.

Percepción de los docentes sobre contenido de la asignatura de Finanzas

El 81,0 \% de los docentes destacan que los contenidos responden a una estructuración adecuada y distribuida en unidades de aprendizaje y de dificultad progresiva.

Los contenidos evaluados en la entrevista a los docentes de los programas de Administración de Empresas arrojaron un promedio de 87,7 \%.

Percepción de los docentes encuestados sobre motivación a los estudiantes

La variable de motivación registró un promedio en baremo del 90,0 \% y una calificación de excelente. Se constató en los docentes encuestados un resultado altamente positivo en lo medianamente y completamente de acuerdo $(85,7 \%)$.

El 85,7 \% de los docentes estimulan la participación, integrando, conduciendo intervenciones, sintetizando, reconstruyendo y desarrollando los temas que vayan surgiendo en la clase. Baremo promedio 90,0 \%. 
Percepción de los docentes encuestados sobre sistema de evaluación

El 90,4 \% de los docentes recogen información de la marcha académica de sus estudiantes orientando y ayudando a superar las dificultades.

En las actividades de autoevaluación, los docentes fueron muy claros en afirmar en un 76,2\% que controlan el aprendizaje de forma progresiva proporcionando retroalimentación inmediata y continua. Baremo promedio $88,0 \%$.

Resultados de las encuestas de desempeño académico de los estudiantes

Percepción de los estudiantes sobre los tipos de metodología recibida

El $88,0 \%$ de los estudiantes entrevistados se acomodaron con el método de talleres teóricos y prácticos. Los estudiantes en el 59,7 \% optaron por el método de juegos financieros. Baremo promedio de $73,1 \%$. Situación que prende alarmas en la didáctica para las finanzas.

Percepción de los estudiantes sobre los contenidos de las asignaturas de Finanzas

Los contenidos del curso de Finanzas sobre calidad, pertinencia, relevancia y la autoría de la fuente de información fueron 85,9 \% positivos según los estudiantes.

En lo relativo al curso de Finanzas en su estructuración adecuada y distribuido en unidades de aprendizajes, los estudiantes en su percepción estuvieron medianamente y completamente de acuerdo con el $77,5 \%$. Baremo promedio $77,8 \%$. 
La motivación de los docentes en el aula y la percepción de los estudiantes

Según los estudiantes los docentes desarrollaron capacidad y empatía para el trabajo colaborativo con equipos interdisciplinarios en un 79,0\%.

El 74,4 \% de los estudiantes encuestados aprobaron medianamente y completamente de acuerdo que los docentes propiciaron procesos de comunicación asertiva. Baremo promedio $77,8 \%$.

Percepción de los estudiantes sobre los currículos de los planes de estudios de AE

El 63,9 \% de los estudiantes conocieron el contenido del curso de Finanzas que estaban desarrollando en el programa universitario.

En la consulta sobre si los créditos que tiene el curso de Finanzas son suficientes para su formación de administrador de empresas, los estudiantes fueron positivos en $71,7 \%$. Baremo promedio $78 \%$.

Percepción de los estudiantes sobre las actividades de aprendizaje en la formación en Finanzas

El 79,8 \% de los estudiantes son positivos con lo planteado en los reglamentos, que establecen que las actividades del aprendizaje deben ser variadas y múltiples, organizadas y secuenciales.

Las actividades de aprendizaje con Baremo de calificación Buena. Promedio de la variable de $79,8 \%$.

Percepción de los estudiantes sobre los sistemas de evaluación de las asignaturas de Finanzas 
Los estudiantes reconocieron que el sistema de evaluación les permitió tener varias alternativas para realizar los trabajos de sus asignaturas de Finanzas mediante foros y grupos de discusión con el 76,4 \%.

Los estudiantes aprobaron con el 74,3 \%, que los docentes propusieron actividades que acumulan información sobre el desarrollo de los cursos orientando y coadyuvando a superar las dificultades.

Confrontación de los resultados de las encuestas de desempeño académico de los estudiantes y docentes de los programas universitarios de Administración de Empresas de las universidades consideradas en la muestra

Confrontación de metodologías entre docentes y la percepción de los estudiantes

En el método talleres teóricos y prácticos se presentaron diferencias de percepciones, siendo más positivos los estudiantes con $88,0 \%$ frente al $80,9 \%$ de los docentes.

En el método colaborativo, las diferencias de percepciones mostraron positivos a los docentes con $76,2 \%$ frente al $61,3 \%$ de los estudiantes.

En el método de casos, los docentes fueron positivos con el 81,0\% frente al 68,6\% de la percepción de los estudiantes.

Confrontación de los contenidos entre docentes y percepción de los estudiantes

En lo referente a la calidad en el sentido de la pertinencia, la relevancia y la autoría de la fuente de información, los resultados de percepción 
mostraron muy favorables a los estudiantes con $85,9 \%$, mientras que los docentes manifestaron acuerdo con el 66,7\%.

La estructuración adecuada de los contenidos, distribuida en unidades de aprendizaje y de dificultad progresiva, se encontró muy favorable en los docentes con 80,9\%, mientras que los estudiantes aprobaron con $77,5 \%$.

Los contenidos en cuanto a un volumen adecuado a las características del grupo y a los objetivos que se persiguen en la consulta de percepción se obtuv que es favorable en los docentes con $66,7 \%$, mientras que para los estudiantes la percepción fue más favorable en 79,5\%.

Confrontación sobre la motivación en el aula entre los docentes y la percepción de los estudiantes

En la motivación sobre propiciar procesos de comunicación asertiva entre los actores educativos, la consulta fue favorable en los docentes con $76,2 \%$, mientras que los estudiantes la aprobaron con el 74,4 \%.

En la consulta sobre motivación en cuanto se favorece el desarrollo del aprendizaje y se da la bienvenida a los estudiantes que participan en el curso, los resultados de confrontación son favorables en los docentes con el $94,4 \%$, mientras que los estudiantes la aprobaron con $83,8 \%$.

Sobre el proceso de animar, estimular la participación integral, conducir intervenciones, sintetizando, reconstruyendo y desarrollando los temas que vayan surgiendo, resultó muy favorable en los docentes con $85,7 \%$, mientras que los estudiantes lo aprobaron en $77,0 \%$. 
Confrontación sobre el sistema de evaluación entre el docente y la percepción de los estudiantes

Sobre la consulta si el docente propone actividades que recogen información de la marcha del estudiante, con el fin de orientarlo y ayudarlo a superar las dificultades que se le pudieran presentar se constató muy favorables en los docentes con el 94,4\%, mientras que los estudiantes la aprobaron con el 74,4\%.

En cuanto a si en la percepción de los créditos del curso de Finanzas son suficientes para la formación del administrador, los docentes fueron poco positivos con el 52,4\%, mientras que los estudiantes la aprobaron con $72,7 \%$.

En cuanto a si en las percepciones sobre currículo, los créditos del curso de Finanzas son suficientes para la formación del administrador los docentes fueron poco positivos con 52,4 \%, mientras que los estudiantes aprobaron con $71,7 \%$.

En las percepciones sobre currículo, el docente realizó evaluación de acuerdo a las reglas de juego -normas-, mediante su adecuación al currículo. Los estudiantes lo aprobaron en un $84,8 \%$, solo el 1,5\% se mostró en desacuerdo.

En la investigación realizada se encontró que los programas de Administración de Empresas del 88,9 \% de los magísteres vinculados no se encontró ningún docente con formación en educación al igual que el $11,1 \%$ de los especialistas.

Se encontraron en la investigación huellas y trazos didácticos en los programas universitarios de Administración de Empresas. Solo los 
programas cuentan con el $44,4 \%$ de docentes de tiempo completo, mientras que el 33,3\% son de medio tiempo y el 22,2\% catedráticos, lo que no garantiza el proceso de diseño educativo.

El 22,2 \% de los docentes vinculados a los programas son investigadores y el 77,8 \% son docentes. Lo que incide en los procesos transversales de la investigación y en la dinámica de la enseñanza-aprendizaje, los contenidos, currículos, actividades del aprendizaje.

La mayoría de las misiones de los programas consultados presentan de manera general los conceptos de innovación, competitividad, pero en cambio las variables globalización, emprendimiento y tecnología de las comunicaciones y de la información no aparecen en las misiones.

Revisada la misión se aprecia un divorcio entre la misión del programa de Administración de Empresas, el plan de estudios y los contenidos curriculares en lo atinente a la didáctica en la formación de finanzas.

Algunas visiones en los programas universitarios analizados son extensas y confusas y no son fáciles de transmitir y explicar, la visión de un programa de Administración de Empresas se encuentra desactualizada porque fue programada al 2015.

Se identificó que los planes de estudio de los programas universitarios de Administración de Empresas son diferenciadores en sus currículos, asignaturas, créditos académicos, los nombres y la ubicación de las asignaturas, el proceso secuencial de las asignaturas, los perfiles profesionales y ocupacionales y la duración de la carrera.

En la actualidad el profesorado se encuentra ante una multiplicación de funciones, desde una carga académica alta, combinada con la investigación, reuniones y comités. 
En próximas investigaciones continuar profundizando, en relación a las dimensiones y componentes de la propuesta para el desarrollo didáctico de los cursos de finanzas sustentados en entornos presenciales y virtuales de enseñanza-aprendizaje y experimentar de manera práctica lo que permitirá perfeccionar la propuesta de este trabajo.

Continuar las investigaciones sobre las dimensiones e indicadores de desempeño académico para la evaluación, selección e implementación de didácticas dinámicas, integradora e innovadoras y estudiar otros posibles entornos que puedan aparecer, así como la evolución de los estudiados en esta investigación.

El administrador del siglo XXI debe tener desarrollado el pensamiento crítico, ser capaz de prevenir y solucionar problemas, de trabajar en grupo, de buscar y analizar información, considerar que el conocimiento por sí solo no es útil y comienza a cobrar utilidad en términos de innovación y transformación solamente cuando se pone al servicio de la sociedad.

Se identificó en el estudio que en la formación en finanzas se destacó como estilo de enseñanza el Nutritivo, después se ubicó en orden de preferencia el estilo Transmisor aplicado por los docentes.

Los tipos de didáctica que utilizan los docentes con mayor aplicación son: la didáctica Comprehensiva con el 85,7 \%, sigue la didáctica Crítica con el 76,2 \%, luego la didáctica Expresiva, la Constructivista, la Expositiva y la didáctica Socrática con el 71,4\%. Es para reflexionar el caso de la didáctica Constructivista que solo se ubicó en el cuarto lugar de ser aplicada por los docentes consultados en la encuesta de investigación. 
El grado de respuesta de los sílabos de finanzas en la estructura curricular para la formación integral de un profesional para la toma de decisiones financiera en un contexto globalizado con aceptación del 81 \%.

En el desarrollo de los sílabos de finanzas se aplicó como metodología común para todos los docentes el método de casos, siguiendo en orden de importancia el método talleres teóricos y prácticos. Este trabajo caracterizó y evidenció que en los tipos de metodologías los estudiantes encuestados manifestaron medianamente y completamente de acuerdo en promedio del 73,1\% que durante el desarrollo de los sílabos de finanzas los docentes aplicaron la metodología del método de casos con el $68,6 \%$.

Se identificó que los estudiantes consultados sobre la pedagogía y currículo manifestaron medianamente y completamente de acuerdo que conocieron el contenido del curso de Finanzas que estaban desarrollando en el programa universitario con el 85,9\%.

Se evidenció que la percepción de los estudiantes sobre la motivación que hacen los docentes de los programas universitarios de Administración fue buena con un promedio del 78,3 \%, según los estudiantes, los docentes desarrollaron capacidad y empatía para el trabajo colaborativo con equipos interdisciplinarios en un 79,0\%.

En las percepciones sobre currículo, el docente realizó evaluación de acuerdo a las reglas de juego -normas-, mediante su adecuación al currículo. Los estudiantes aprobaron el currículo en un $84,8 \%$, solo el $1,5 \%$ se mostró en desacuerdo.

En cuanto a si en las percepciones sobre currículo, los créditos del curso de Finanzas son suficientes para la formación del administrador 
los docentes fueron positivos poco con $52,4 \%$, mientras que los estudiantes aprobaron con $71,7 \%$.

En las percepciones al sistema de evaluación, el docente aplicó diversas estrategias de evaluación que permitieron al alumno participar en el desarrollo de trabajos, foros o grupos de discusión y colaboración con los compañeros, fueron positivas por los docentes con $76,2 \%$, mientras que los estudiantes aprobaron con 76,4\%.

En las percepciones motivacionales se favorece el desarrollo del aprendizaje y da la bienvenida a los estudiantes, muy favorables por los docentes con $94,4 \%$, mientras que los estudiantes aprobaron con $83,8 \%$.

\section{Recomendaciones}

- Sugerir la generalización de la propuesta de desarrollo de una Didáctica Dinámica en Contexto (DDC) a otras facultades de las universidades tomadas en la muestra, así como a otras instituciones de educación superior del país como una posible vía para perfeccionar los niveles alcanzados en la formación en finanzas sobre la base de una didáctica dinámica integradora e innovadora.

- Realizar acciones encaminadas a la socialización de la propuesta de desarrollo didáctico dinámico teórico-práctico para el diseño de los cursos presenciales y virtuales en el contexto de la capacitación de los profesores que laboran en las universidades tomadas en la muestra y en otras del país de tal manera, que sea valorada, analizada, discutida y enriquecida $y$, de ser posible, aplicada por los mismos.

En próximas investigaciones continuar profundizando, en relación a las dimensiones y componentes de la propuesta para el desarrollo di- 
dáctico dinámico de los cursos de finanzas sustentados en entornos presenciales y virtuales de enseñanza-aprendizaje y experimentar de manera práctica lo que permitirá perfeccionar la propuesta de este trabajo.

Continuar las investigaciones sobre las dimensiones e indicadores de desempeño académico para la evaluación, selección e implementación de didácticas dinámicas, integradoras e innovadoras y estudiar otros posibles entornos que puedan aparecer, así como la evolución de los estudiados en esta investigación.

Los resultados alcanzados contribuyen desde el punto de vista teórico con el concepto de entorno de enseñanza-aprendizaje desde una perspectiva constructivista. Además, desarrolla la instrumentación de acciones concretas para elevar la preparación pedagógica, didáctica y técnica de los profesores de Administración de Empresas, de otras facultades, de la propia universidad y de otros centros universitarios, así como de los profesionales que se preparan en las carreras de referencia. De igual manera, revisados los modelos pedagógicos y dados los resultados del trabajo doctoral realizado se presenta la propuesta de un desarrollo didáctico dinámico en contexto para la formación en finanzas con fundamento en el modelo pedagógico consciente, dinámico e innovador.

Apoyar a los programas académicos de Administración de Empresas en los nuevos diseños curriculares para ajustar los contenidos de las asignaturas de Gerencia financiera y Análisis financieros para que los estudiantes del área financiera desarrollen sus capacidades y competencias.

Con la propuesta Didáctica Dinámica en Contexto (DDC) en la forma- 
ción en finanzas desde la epistemología, la didáctica y la determinación de los contenidos curriculares de la enseñanza de las finanzas, se intenta ayudar a:

- Los docentes universitarios de Finanzas, a actualizar los contenidos, métodos, prácticas y modos de enseñar los conocimientos financieros.

- Los estudiantes de Finanzas, a lograr una mejor formación en la disciplina, que les permita comprender, interpretar y dar respuesta a los distintos problemas y situaciones que configuran una decisión financiera para ser mejor cualificados en el área financiera.

- Transformar la enseñanza, ya que el currículo de las asignaturas financieras, visto como una síntesis de posiciones epistemológicas, de abordajes didácticos y con unos determinados contenidos conceptuales, procedimentales y actitudinales, es lo que determina lo que pasa en el aula entre profesores y estudiantes.

La propuesta Didáctica Dinámica en Contexto (DDC) constituye una exigencia para lograr los resultados deseados en materia de desarrollo educativo, tecnológico y transferencia de conocimiento, ya que si bien la propuesta nos coloca en la senda correcta, el camino es aún muy largo por recorrer en la vida académica, se demanda una reforma en los diseños de las asignaturas para facilitar las actividades de desarrollo didáctico, tecnológico y transferencia de conocimiento de las IES y otras entidades, conformando así una masa innovadora de instituciones educativas y de investigadores con capacidad para incursionar de manera activa en el creciente mercado educativo competitivo, que no solo es el que puede tener la iniciativa, sino que eventualmente tendrá bajo su responsabilidad algo que ahora promueve la propia ley de manera explícita: la aplicación y comercialización de la innovación didáctica. 
Con lo anterior, se pretende elevar los niveles de desarrollo científico y tecnológico de lo didáctico e incidir en el desarrollo económico, de manera tal que las acciones de la didáctica dinámica y la transferencia de conocimiento aporten de manera significativa al desarrollo educativo de este país, y en retorno, las actividades de investigación y desarrollo lleguen lo antes posible a significar ese anhelado $1 \%$ del PIB.

\section{REFERENCIAS BIBLIOGRÁFICAS}

Aebli, H. (2000). Las 12 formas básicas de enseñar, una didáctica basada en la psicología. España: Narcea.

Aguilar, N. Á. (14 de octubre de 2004). Disponible en: http://sisomartinezinicial.blogspot.com/. Obtenido de Didáctica Inicial de Siso Martínez. Recuperado el 03 de 02 de 2013.

Attala, A. (2009). La enseñanza de finanzas en las universidades de Argentina. Buenos Aires: Universidad de Buenos Aires.

Bachelard, G. (1985). Materialismo Racional. Buenos Aires: Paidós.

Basabe, S. (2013). Desempeño del docente en las aulas virtuales. Mérida: Producciones Editoriales C.A.

Bernstein, B. (1971). Hacia una teoría del discurso pedagógico. Pedagogía y Educación, 47.

Correa, C. (2013). Seminario de tesis doctoral. Pedagogía y Educación. Objeto de investigación científica en la formación doctoral. Barranquilla. Chávez, N. (2001). Introducción a la investigación educativa. Maracaibo: Universal.

De Zubiría, J. (2001). Los modelos pedagógicos. Bogotá: Fondo de Publicaciones Alberto Merani.

Dewey, J. P. (2004). Teoría de la valoración. México: Siruela.

Díaz, D. (1999). La didáctica universitaria: Referencia imprescindible para una enseñanza de calidad. Revista Electrónica Interuniversitaria de formación del profesorado, 108-116.

Eigenmann, M. (1981). Desarrollo secuencial del currículum. Madrid: Anaya. 
Galeano, M. (2004). Diseño de proyectos con la investigación. Medellín: Universidad de Medellín.

García, M. (1969). Didáctica general. Madrid: Ediciones Anaya.

Gitman, L. (2000). Fundamentos de Administración Financiera. México: Prentice-Hill.

Gómez, A. (2010). Lógica y conjuntos en la enseñanza universitaria del Caribe colombiano: 1961-2000. (Tesis doctoral). Cartagena: Universidad de Cartagena.

Hernández, A. (2014). Metodologica de la investigación. México: McGraw-Hill.

Klafki, W. (1954). Stufen des pädagogischen Denkens. Suiza: Bildung und Erziehung,.

Martínez, M. (2007). Paradigma Emergente. México: Trillas.

Monetti, E. (2015). La didáctica de las cátedras universitarias, estilo de enseñanzas y planificación de clases. Buenos Aires: Colección Universidad.

Mora, R. (2014). Currículo y saberes en la formación de abogados. Barranquilla: Ediciones Universidad Simón Bolívar.

Ontoria, A. G. (2003). Potenciar la capacidad de aprender a aprender. México: Alfaomega.

Ossa, A. (2013). Módulo Tendencias curriculares contemporáneas. (Doctorado en Ciencias de la Educación). Cartagena, Bolívar, Colombia: Universidad Simón Bolívar.

Philippatos, G. (1974). Fundamentos de administración financiera. Bogotá: Editorial McGraw-Hill.

Puche, R. R. (2010). Administración Financiera Global (primera edición ed., Vol. 1). (U. S. Bolívar, Ed.). Barranquilla, Atlántico, Colombia: Artes Gráficas Industriales Ltda.

Ramírez, J. (14 de 04 de 2013). La psicopedagogía. Obtenido de: Psicopedagogia.com.definicion/didactica.

Rico, F. (2013). Los premios Nobel de Economía (1969-2013). Desarrollo Indoamericano, 122, 36-65.

Rico, F. (2016). Tesis doctoral Didáctico para la formación en Finanzasen 


\section{Didáctica para la formación en finanzas en programas}

universitarios de Administración de Empresas

programas de Adiministración de Empresas. Barranquilla: Universidad Simón Bolívar.

Sinisterra, G. P. (2001). Contabilidad, sistema de información para las organizaciones. Bogotá: Editorial McGraw-Hill.

Stenhouse, L. (1987). Investigación y desarrollo del currículum. Madrid: Morata.

Suárez, M. (1993). Las corrientes pedagógicas contemporáneas y sus implicaciones en las tareas del docente y en el desarrollo curricular. Táchira, Venezuela: Universidad de los Andes.

Tamayo y Tamayo (2001). El proceso de la investigación cientifica. México: Limusa.

Tyler, R. (1949). Basic Principles of Curriculum Development. Chicago: Universidad de Chicago.

Van Horne, J. (1988). Fundamentos de Administración Financiera. México: Editorial Prentice-Hill.

Zambrano, A. (1963). Formación, experiencia y saber. Bogotá: Editorial Magisterio.

Zambrano, A. (2002). Los hilos de la palabra: Pedagogía y Didáctica. (Primera ed., Vol. 2). Cali, Valle del Cauca: Artes Gráficas del Valle. Zambrano, N. (2005). Estrategias didácticas para la enseñanza de los diferentes métodos y técnicas aplicadas en la praxis de la Ingeniería Industrial, desde una perspectiva constructivista. Venezuela: Universidad Nacional Abierta de Venezuela.

Zambrano, A. (2006). Tres tipos de saber en la formación del maestro. Memorias de la conferencia central. II Encuentro Nacional de Escuelas Normales, sujetos, contenidos y contexto (pág. 17). Popayán: Secretaría de Educación del Valle del Cauca.

Cómo citar este capítulo:

Rico-Calvano, F. (2017). Didáctica para la formación en finanzas en programas universitarios de Administración de Empresas. En Prieto-Pulido, R. \& Palacios-Arrieta, A. (2017). Gestión contable y financiera en las organizaciones. Un enfoque global. Ediciones Universidad Simón Bolívar. Barranquilla-Colombia. 121-151. 